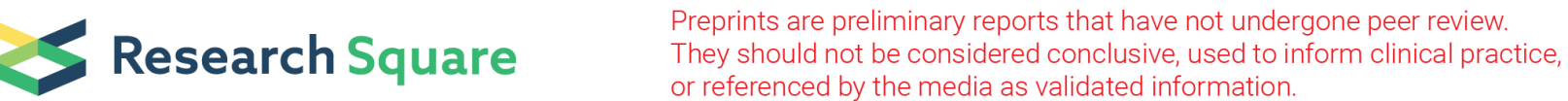

\section{Early permanent cerebrospinal fluid diversion lowers the rate of nosocomial meningitis in aneurysmal subarachnoid hemorrhage}

\section{Davide Marco Croci}

Universitatsspital Basel

Martina Dalolio

Universitatsspital Basel

Soheila Aghlmandi

Universitatsspital Basel

Ethan Taub

Universitatsspital Basel

\section{Daniel Zumofen}

Universitatsspital Basel

\section{Raphael Guzman}

Universitatsspital Basel

\section{Luigi Mariani}

Universitatsspital Basel

Michel Roethlisberger ( $\square$ michel.roethlisberger@usb.ch )

Universitatsspital Basel https://orcid.org/0000-0003-0932-0193

\section{Research}

Keywords: External ventricular drain, infections, ventriculo-peritoneal shunting, cerebrospinal fluid diversion, nosocomial meningitis, cerebral vasospasm, delayed cortical infarction, aneurysmal subarachnoid hemorrhage

Posted Date: January 21st, 2020

DOI: https://doi.org/10.21203/rs.2.21401/v1

License: (1) (1) This work is licensed under a Creative Commons Attribution 4.0 International License.

Read Full License 


\section{Abstract}

Background: Early permanent cerebrospinal fluid (CSF) diversion for hydrocephalus after aneurysmal subarachnoid hemorrhage (aSAH) might shorten the duration of external ventricular drainage (EVD) and thereby reduce infectious complications. The potential effect on the rate of delayed cerebral vasospasm (DCVS) and associated morbidity has not been studied to date. The objective of this study was to detect any association with EVD-associated infections (EVDAI), symptomatic DCVS, or delayed cerebral ischemia ( $\mathrm{DCl})$ by the time of hospital discharge.

Methods: A single-center dataset of aSAH patients who received a permanent CSF diversion procedure between 2009 and 2018 was used for the evaluation. The subjects were divided into an "early group" if such a procedure was performed up to 14 days after the ictus, and a "late group" if it was performed from the 15 th day onward. The statistical analysis employed univariable and multivariable logistic regression models.

Results: Among 274 consecutive aSAH patients, 39 (14.2\%) had a permanent CSF diversion procedure. While the blood clot burden was similarly distributed, patients with early permanent CSF diversion (20 out of $39,51.2 \%$ ) had higher levels of consciousness on admission. Early permanent CSF diversion was associated with a shorter duration of EVD (OR $0.73,95 \% \mathrm{Cl} 0.58-0.92$ per day). Higher catheter colonization to EVDAl ratio (1/7 out of 20 vs. $7 / 7$ out of 19$)$ and a markedly lower frequency of EVDAI (OR $0.08,95 \% \mathrm{Cl} 0.01-0.80$ ) were detected. The prevalence ( 5 vs. 37 ) and the cumulative incidence ( 3 vs. 18) of EVDAI were remarkably lower in patients receiving early permanent CSF diversion. The occurrence of CSF-diversion device obstruction, the rate of symptomatic DCVS (OR $0.61,95 \% \mathrm{Cl} 0.16-2.27)$ or detected $\mathrm{DCl}$ on computed tomography (OR $0.35,95 \% \mathrm{Cl} 0.08-1.47$ ), and the likelihood of a poor outcome at discharge did not differ between the two groups (OR $0.88,95 \% \mathrm{Cl} 0.24-3.22$ ).

Conclusions: Early permanent CSF diversion in good grade aSAH patients is associated with a shorter duration of EVD, lower catheter colonization rates, and fewer infectious complications. The timing of permanent CSF diversion had no detectable effect on DCVS-related morbidity. These findings need to be confirmed in larger cohorts.

\section{Background}

The potential adverse sequelae of acute aneurysmal subarachnoid hemorrhage (aSAH) are well known and have a crucial impact on morbidity and mortality due to this disease [1].

Acute hydrocephalus arises in approximately one third of patients and is treated with temporary cerebrospinal fluid (CSF) diversion in 6 to $30 \%$ [2, 3]. Prolonged external ventricular drainage (EVD) is associated with infection (EVDAl), with an incidence of 11.4 per 1000 catheter-days in the first two weeks [4]. Various risk factors for EVDAI have been identified $[5,6]$. The duration of external drainage and the presence and extent of subarachnoid and intraventricular blood are of special importance for the development of infectious complications [4-7]. Nowadays, EVDAl is considered to be one of the most 
important nosocomial infections that negatively influence the neurological outcome of aSAH patients. Acknowledged mechanisms behind this clinical observation are the exacerbation of inflammatory processes, thrombogenicity, and disturbances of CSF-dynamics [8-14]. According to the literature, between $8 \%$ and $63 \%$ of aSAH patients eventually undergo a permanent CSF diversion (shunting) procedure $[3,15]$. Factors associated with the latter are high blood clot burden, acute hydrocephalus, nosocomial infections, re-hemorrhage, posterior location of the ruptured aneurysm, and age $\geq 60$ years [3, $10,16,17]$.

Symptomatic delayed cerebral vasospasm (DCVS) after aSAH mostly occurs on days 3 to 14 after the ictus; it is associated with delayed cerebral ischemia (DCl) and with increased morbidity and mortality [18]. Neurosurgeons generally prefer to perform permanent CSF diversion procedures after the DCVS phase $[19,20]$. There is a legitimate concern that surgical procedures requiring patient transfer, general anesthesia, and intensified sedation, with potential intraoperative hemodynamic disturbances, might increase the risk of cerebral hypoperfusion and thus of DCVS and DCI [20-24]. Furthermore, it is assumed that a higher frequency of permanent CSF diversion device obstruction caused by the circulating blood occurs in early stages of the disease $[25,26]$.

Only a few publications to date have concerned early, permanent CSF diversion in aSAH [27, 28]. Early EVD weaning with early permanent CSF diversion (if necessary) could be an effective treatment for SAHinduced hydrocephalus in patients with severe SAH [27]. On the whole, there is no evidence on whether early permanent CSF diversion in aSAH carries a higher risk of DCVS and DCl. There is likewise uncertainty over the question whether the achieved shorter period of extracorporeal diversion is associated with a lower infection rate $[3,4,10,15,20-22]$. In sum, the purpose of this cohort study was to determine the rate of CVS- and catheter-related infectious complications of aSAH patients who had undergone a permanent CSF diversion procedure within 14 days of the ictus versus at later times, when the risk of DCVS-related morbidity is much lower.

\section{Methods}

The present cohort was extracted from a single-center data set of aSAH patients. The assessment, treatment, and follow-up were performed according to center-specific standard procedures for patients with aSAH. Institutional Review Board (IRB) and local ethics committee (EKNZ, Basel, Switzerland) approval was obtained and accredited (Project-ID 2018-02129). The ethics committee waived the requirement for written informed consent (justification: disproportionality) for patients recruited prior to 2014, when the new Swiss Human Research Act went in force. As of 2014, written informed consent was obtained from all participants.

Study design: This is a cohort study retrospectively collected in the year 2009 and prospectively collected in the years 2010-2018. The dataset was anonymized consecutively for analysis. Because of the purely observational nature of the study, no registration in a trial registry was necessary. 
Study center: All patients within the present data set were treated at the University Hospital of Basel, an accredited tertiary neurovascular center.

Study population: Data were collected from all patients admitted to the study center with aSAH from a documented ruptured intracranial aneurysm. Patients with non-aneurysmal SAH and patients who died on the day of admission were excluded.

Data collection: A set of variables of interest was predefined. These variables were from hospital charts and pooled in a secured, local registry. A recollection of EVD-related variables and infectious complications as well as CSF-laboratory investigations was conducted in 2016.

Study variables: For the present study, the following variables were extracted from the local data set: patient characteristics (age, sex); aSAH specific admission scores (Glasgow Coma Scale [GCS] [29], World Federation of Neurosurgical Societies [WFNS] score [30]); radiological characteristics (Fisher grade [31], Barrow Neurological Institute (BNI) scale [32], presence of intraventricular or intracerebral hemorrhage, presence of acute hydrocephalus [occurring within 72 hours after ictus]) [16]; aneurysm characteristics (location of the ruptured aneurysm and maximal aneurysm diameter treatment modality [open surgical clipping or endovascular treatment]); information on temporary and permanent CSF diversion procedures (time point of implantation, duration of external drainage, weaning period, time point of conversion to a permanent CSF diversion); infectious complications (EVDAI); laboratory parameters of infections such as leucocyte count, proteins, and hemoglobin measured in CSF and blood); information on DCVS and DCI (time of onset, clinical or radiological manifestation, treatment); outcome variable (modified Rankin scale [mRS] [33] and in-hospital death); and duration of hospital stay.

The indication for external ventricular drainage and the timing of EVD weaning were at the treating neurosurgeon's discretion. Generally, the weaning process consisted of incremental raising of the extracorporeal CSF diversion system for 48 hours, followed by drain closure for 24 hours. Unsuccessful weaning was defined as either clinical manifestation of hydrocephalic symptoms or radiological demonstration of ventricular enlargement on a CT scan within this period.

Cases of EVDAl were confirmed in accordance with the modified criteria for nosocomial infections of the Centers for Disease Control and Prevention (CDC) [34]. A positive culture of CSF or a ventricular catheter tip without other abnormal CSF findings or clinical signs suggesting infection was designated as colonization [9, 34]. EVDAI was diagnosed if a positive culture of the CSF or catheter tip was present and accompanied by further pathological CSF findings or at least one new sign or symptom of CNS infection in the absence of any other known cause [9, 34]. EVDAl was also diagnosed in some cases with negative cultures in which there were pathological CSF findings and/or clinical manifestations of infection and antimicrobial therapy was given $[9,34]$.

DCVS was defined as local vasospasm visible on computed tomographic (CT) angiography or digital subtraction angiography accompanied by neurological worsening for at least 2 hours [35]. DCl was 
defined according to the multidisciplinary research group criteria [36] as brain infarction visible on CT or magnetic resonance imaging in the first 6 weeks after SAH.

Primary and secondary endpoints: The primary endpoint was the occurrence of EVDAI. The secondary endpoints were the occurrence of symptomatic or DCVS, the presence of a stroke on the last CT obtained before hospital discharge defined as $\mathrm{DCl}$, and a poor mRS score (4-6) at discharge, or in-hospital death.

Follow-up: To assure the availability and consistency of data for statistical analysis, we only collected standardized variables (occurrence of EVDAI and DCVS) during hospitalization. Unambiguous time points were defined, e.g. the day before a permanent CSF diversion procedure (CSF sampling) and patient discharge ( $\mathrm{DCl}, \mathrm{mRS}$, in-hospital death). A detailed patient inclusion profile including the number of participants with available data at each time point is provided in Fig. 1.

Statistical analysis: We provide descriptive statistics for a set of predefined variables of interest by reporting means and $95 \%$ confidence intervals, or median and interquartile ranges for continuous variables, as appropriate. For categorical variables, counts and percentages were reported. The patients were divided into two groups: the "early group," in which a permanent CSF diversion procedure was performed $\leq 14$ days after the ictus, and the "late group," in which it was performed $>14$ days after the ictus. To obtain the association between the outcome of interest and important variables (known to be associated with temporary and permanent CSF diversion or influence defined outcomes), the logistic regression model was used. The variables used in the univariable model(s) are dichotomized age („higher age" [age $\geq 60$ ] vs. "lower age" [age < 60]), BNI scale ("high BNI scale” [BNI 4-5] vs. "low BNI scale" [BNI 1-3]), WFNS ("low WFNS" [WNFS 1-3] vs. "high WFNS [WNFS 4-5]), and mRS ("Good mRS” [mRS 1-3] vs. "Poor mRS" [mRS 4-6]). The duration of external ventricular drainage (in days) was used as a continuous variable. Risk factors that had more than $10 \%$ increase or decrease in OR (OR $\leq 0.9$ or $\geq 1.10)$ and which were considered clinically relevant for the endpoints of interest were included in the multivariable model (see additional Tables I-III; Additional file 1). Due to the small size of this cohort and also the low number of events, a maximum of three variables was included in the multivariable model(s). The duration of external ventricular drainage and late permanent CSF diversion are not mutually exclusive variables and were analyzed separately. Measures of accuracy were calculated for the sample estimates with bootstrapping statistics. Confidence intervals were calculated with the profile likelihood method based on the Wald test statistic. Statistical significance was set at $P \leq .05$. Statistical analyses were performed in SPSS (IBM SPSS Statistics 22, 2013, New York, USA).

\section{Results}

Patient characteristics: The data set for the years 2009 to 2018 comprises data on 274 aSAH patients, of whom 39 (14.2\%) patients had undergone permanent CSF diversion and were eligible for analysis. 20 (51\%) of these patients had received a permanent CSF diversion up to 14 days after the ictus (early group), and 19 (49\%) had received a permanent CSF diversion 15 days or more after the ictus (late group). The procedure of choice was a ventriculoperitoneal shunt with an adjustable valve (Codman- 
Hakim ${ }^{\circledR}$, Codman \& Shurtleff, Raynham, USA). Sex and mean age were similarly distributed between the two groups. Patients receiving an early permanent CSF-diversion had a substantially higher level of consciousness, measured by the GCS- and WFNS score on admission. The radiological blood clot burden, represented by the Fisher-Grade and the BNI-score, as well as the presence of intraventricular blood on admission, did not differ between the groups (Table 1). 
Table 1

Baseline characteristics of 39 out of 274 aSAH patients treated with permanent CSF diversion

\begin{tabular}{|c|c|c|}
\hline Baseline Characteristics & $\begin{array}{l}\text { Early permanent CSF } \\
\text { diversion ( } \leq 14 \text { days) }\end{array}$ & $\begin{array}{l}\text { Late permanent CSF } \\
\text { diversion ( } \geq 15 \text { days) }\end{array}$ \\
\hline & $\mathrm{n}=20$ & $\mathrm{n}=19$ \\
\hline Sex & $\mathrm{n}(\%)$ & $\mathrm{n}(\%)$ \\
\hline Female & $12(60)$ & $9(47.4)$ \\
\hline Age & Mean $( \pm S D)$ & Mean $( \pm S D)$ \\
\hline Years & $60( \pm 13)$ & $60( \pm 8)$ \\
\hline \multicolumn{3}{|c|}{ Clinical information on admission } \\
\hline GCS score & Median & Median \\
\hline Level of consciousness & 13 & 3 \\
\hline WFNS score & $\mathrm{n}(\%)$ & $\mathrm{n}(\%)$ \\
\hline $1-2$ & $10(50)$ & $4(21)$ \\
\hline $3-4$ & $4(20)$ & $4(21)$ \\
\hline 5 & $6(30)$ & $11(58)$ \\
\hline Focal neurological deficit & $\mathrm{n}(\%)$ & $\mathrm{n}(\%)$ \\
\hline Present on admission & $3(15)$ & $4(21)$ \\
\hline Cranial nerve deficit & $\mathrm{n}(\%)$ & $\mathrm{n}(\%)$ \\
\hline Present on admission & $2(10)$ & $5(26)$ \\
\hline \multicolumn{3}{|c|}{$\begin{array}{l}\text { Radiological information (computed } \\
\text { tomography scan) on admission }\end{array}$} \\
\hline Fisher Grade & $\mathrm{n}(\%)$ & $\mathrm{n}(\%)$ \\
\hline 3 & $5(25)$ & $2(11)$ \\
\hline 4 & $15(75)$ & $17(90)$ \\
\hline BNI Score & $n(\%)$ & $\mathrm{n}(\%)$ \\
\hline $2-3$ & $5(25)$ & $6(32)$ \\
\hline 4 & $9(45)$ & 7 (37) \\
\hline
\end{tabular}

n: number; SD: standard deviation; CSF: cerebro-spinal fluid; GCS: Glasgow Coma Scale; WNFS: World Federation of Neurological Societies; BNI: Barrow Neurological Institute 


\begin{tabular}{|c|c|c|}
\hline Baseline Characteristics & $\begin{array}{l}\text { Early permanent CSF } \\
\text { diversion ( } \leq 14 \text { days) }\end{array}$ & $\begin{array}{l}\text { Late permanent CSF } \\
\text { diversion ( } \geq 15 \text { days) }\end{array}$ \\
\hline 5 & $6(30)$ & $6(32)$ \\
\hline Intraventricular hemorrhage & $\mathrm{n}(\%)$ & $\mathrm{n}(\%)$ \\
\hline Present on admission & $15(75)$ & $17(90)$ \\
\hline Intraparenchymal hemorrhage & $\mathrm{n}(\%)$ & $\mathrm{n}(\%)$ \\
\hline Present on admission & $1(5)$ & $1(6)$ \\
\hline Acute hydrocephalus (<72 h) & $\mathrm{n}(\%)$ & $\mathrm{n}(\%)$ \\
\hline Present on admission & $19(95)$ & $17(90)$ \\
\hline \multicolumn{3}{|c|}{ Ruptured aneurysm characteristics } \\
\hline Aneurysm site & $\mathrm{n}(\%)$ & $\mathrm{n}(\%)$ \\
\hline Anterior circulation & $18(90)$ & $15(79)$ \\
\hline Posterior circulation & $1(5)$ & $4(21)$ \\
\hline Missing information & $1(5)$ & $0(0)$ \\
\hline Aneurysm size $e^{\star \star}$ & Mean $( \pm S D)$ & Mean $( \pm S D)$ \\
\hline Maximal diameter (mm) & $7( \pm 3)$ & $6( \pm 4)$ \\
\hline Aneurysm treatment & $\mathrm{n}(\%)$ & $\mathrm{n}(\%)$ \\
\hline Surgical clipping & $3(15)$ & $2(11)$ \\
\hline Endovascular treatment & $16(80)$ & $16(84)$ \\
\hline Missing information & $1(5)$ & $0(0)$ \\
\hline \multicolumn{3}{|c|}{$\begin{array}{l}\text { n: number; SD: standard deviation; CSF: cerebro-spinal fluid; GCS: Glasgow Coma Scale; WNFS: World } \\
\text { Federation of Neurological Societies; BNI: Barrow Neurological Institute }\end{array}$} \\
\hline
\end{tabular}

Temporary extra-corporal CSF diversion: 36 of the 39 patients (92.3\%) had an acute onset of hydrocephalus within 72 hours of their aSAH. A temporary CSF diversion with a Silverline ${ }^{\circledR}$ catheter (Spiegelberg, Hamburg, Germany) was performed in all patients on the day the hydrocephalus was diagnosed. The groups did not differ with respect to the presence of acute hydrocephalus, unsuccessful EVD weaning, and late-onset hydrocephalus after the weaning period (Table 2). As expected, the duration of EVD was significantly lower in the early group, and this association was confirmed in the univariable and the multivariable logistic regression analysis (Table 3, Fig. 2A and B). 
Table 2

Outcome table comparing primary and secondary outcomes in 39 patients with aSAH who underwent either early or late permanent CSF diversion. The positive occurrence of a colonized catheter per 100 days is reported. The cumulative incidence of EVDAI was approximated by the ratio of positive to evaluable cases. A positive culture of the CSF or drain device without CSF findings or clinical signs suggesting central nervous system infection was designated as colonization of the external CSF drain device. Positive cases of catheter colonization were defined as colony-forming unit (CFU) count above the lower limit of quantification.

\begin{tabular}{|c|c|c|c|}
\hline & $\begin{array}{l}\text { Early permanent CSF } \\
\text { diversion ( } \leq 14 \text { days) }\end{array}$ & $\begin{array}{l}\text { Late permanent CSF } \\
\text { diversion ( } \geq 15 \text { days) }\end{array}$ & $\begin{array}{l}\text { Qualitative } \\
\text { analysis }\end{array}$ \\
\hline Temporary CSF diversion & $n=20$ & $n=19$ & \\
\hline Time course of EVD & Mean $( \pm S D)$ & Mean $( \pm S D)$ & $p$-value \\
\hline EVD insertion after ictus (days) & $0.1( \pm 0.2)$ & $0.4( \pm 0.5)$ & - \\
\hline Total days with EVD & $11( \pm 2)$ & $17( \pm 9)$ & $0.002 \S$ \\
\hline EVD weaning & $\mathrm{n}(\%)$ & $\mathrm{n}(\%)$ & \\
\hline Unsuccessful EVD-weaning & $16(80)$ & $13(68)$ & $0.480^{\#}$ \\
\hline $\begin{array}{l}\text { Hydrocephalus after EVD- } \\
\text { weaning }\end{array}$ & $5(25)$ & $2(11)$ & $0.408^{\#}$ \\
\hline Catheter colonization & $\mathrm{n}(\%)$ & $\mathrm{n}(\%)$ & \\
\hline $\begin{array}{l}\text { Positive events per evaluable } \\
\text { case }\end{array}$ & $7(35)$ & $7(37)$ & - \\
\hline $\begin{array}{l}\text { Positive event occurrence (per } \\
100 \text { days) }\end{array}$ & 50 & 50 & - \\
\hline \multicolumn{4}{|l|}{ EVD-associated infection } \\
\hline $\begin{array}{l}\text { Positive events per evaluable } \\
\text { case }\end{array}$ & $1(5)$ & $7(37)$ & $0.020 \#$ \\
\hline Prevalence (per 100 persons) & 5.0 & 36.9 & - \\
\hline $\begin{array}{l}\text { Incidence rate per EVD day in } \\
\text { situ (\%) }\end{array}$ & 0.5 & 2.1 & - \\
\hline Cumulative incidence (\%) & 2.6 & 17.9 & - \\
\hline \multicolumn{4}{|l|}{ Permanent CSF diversion } \\
\hline \multicolumn{4}{|c|}{ 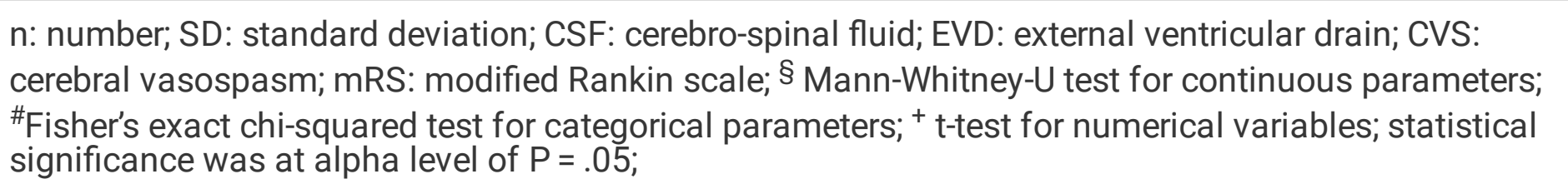 } \\
\hline
\end{tabular}




\begin{tabular}{|c|c|c|c|}
\hline & $\begin{array}{l}\text { Early permanent CSF } \\
\text { diversion ( } \leq 14 \text { days) }\end{array}$ & $\begin{array}{l}\text { Late permanent CSF } \\
\text { diversion ( } \geq 15 \text { days) }\end{array}$ & $\begin{array}{l}\text { Qualitative } \\
\text { analysis }\end{array}$ \\
\hline Time-point of implantation & Mean $( \pm S D)$ & Mean $( \pm S D)$ & \\
\hline Days after ictus & $12( \pm 2)$ & $21( \pm 6)$ & $<0.001^{+}$ \\
\hline $\begin{array}{l}\text { CSF values at day of } \\
\text { implantation }\end{array}$ & Mean $( \pm S D)$ & Mean $( \pm S D)$ & \\
\hline Total Protein (mg/L) & $542( \pm 282)$ & $735( \pm 445)$ & $0.115^{+}$ \\
\hline $\begin{array}{l}\text { Adjusted total leucocytes } \\
(\mathrm{x} 10 \mathrm{e} 6 / \mathrm{L})\end{array}$ & $123( \pm 159)$ & $30( \pm 27)$ & $0.119^{+}$ \\
\hline Lactate (mmol/L) & $3( \pm 1)$ & $3( \pm 1)$ & $0.796^{+}$ \\
\hline Glucose (mmol/L) & $3( \pm 1)$ & $3( \pm 1)$ & $0.951^{+}$ \\
\hline Red blood cell count (x10e6/L) & $11544( \pm 19473)$ & $6242( \pm 9401)$ & $0.325^{+}$ \\
\hline $\begin{array}{l}\text { Complications } 1 \text { months after } \\
\text { implantation }\end{array}$ & $n(\%)$ & n (\%) & \\
\hline Obstruction & $1(5)$ & $1(6)$ & - \\
\hline Infectious complications & $1(5)$ & 00 & - \\
\hline $\begin{array}{l}\text { Malposition of CSF diversion } \\
\text { device tip }\end{array}$ & $1(5)$ & $1(6)$ & - \\
\hline \multicolumn{4}{|l|}{$\begin{array}{l}\text { Delayed cerebral vasospasms } \\
\text { (DCVS) }\end{array}$} \\
\hline Confirmation and treatment & $n(\%)$ & n (\%) & \\
\hline $\begin{array}{l}\text { Radiological and clinical } \\
\text { confirmation }\end{array}$ & $8(40)$ & $10(53)$ & $0.527^{\#}$ \\
\hline $\begin{array}{l}\text { Onset before permanent CSF } \\
\text { diversion }\end{array}$ & $3(15)$ & $10(53)$ & $0.019 \#$ \\
\hline $\begin{array}{l}\text { Onset after permanent CSF } \\
\text { diversion }\end{array}$ & $5(25)$ & $0(0)$ & $0.047^{\#}$ \\
\hline Endovascular chemical dilation & $5(25)$ & $5(26)$ & - \\
\hline Endovascular balloon dilation & $1(5)$ & $3(16)$ & - \\
\hline
\end{tabular}

n: number; SD: standard deviation; CSF: cerebro-spinal fluid; EVD: external ventricular drain; CVS: cerebral vasospasm; mRS: modified Rankin scale; $§$ Mann-Whitney-U test for continuous parameters; \#Fisher's exact chi-squared test for categorical parameters; ${ }^{+} t$-test for numerical variables; statistical significance was at alpha level of $P=.05$; 


\begin{tabular}{|c|c|c|c|}
\hline & $\begin{array}{l}\text { Early permanent CSF } \\
\text { diversion ( } \leq 14 \text { days) }\end{array}$ & $\begin{array}{l}\text { Late permanent CSF } \\
\text { diversion ( } \geq 15 \text { days) }\end{array}$ & $\begin{array}{l}\text { Qualitative } \\
\text { analysis }\end{array}$ \\
\hline Time course & Mean $( \pm S D)$ & Mean $( \pm S D)$ & \\
\hline Days of DCVS onset after ictus & $12( \pm 6)$ & $8.5( \pm 4)$ & $0.131^{\S}$ \\
\hline $\begin{array}{l}\text { Days of DCVS onset before } \\
\text { permanent CSF diversion }\end{array}$ & $4( \pm 3)$ & $14( \pm 7)$ & - \\
\hline $\begin{array}{l}\text { Days of DCVS onset after } \\
\text { permanent CSF diversion }\end{array}$ & $2( \pm 3)$ & - & - \\
\hline \multicolumn{4}{|l|}{ Delayed cortical infarcts (DCI) } \\
\hline Confirmation & $\mathrm{n}(\%)$ & $n(\%)$ & \\
\hline $\begin{array}{l}\text { Presence of a stroke in } \\
\text { computed tomography at } \\
\text { discharge }\end{array}$ & $4(20)$ & $10(53)$ & $0.048^{\#}$ \\
\hline \multicolumn{4}{|l|}{$\begin{array}{l}\text { Morbidity and mortality at } \\
\text { discharge }\end{array}$} \\
\hline Duration of hospitalization & Mean $( \pm$ SD) & Mean $( \pm$ SD) & \\
\hline Days (admission to discharge) & $23.5( \pm 7)$ & $29( \pm 7)$ & $0.057 \S$ \\
\hline $\begin{array}{l}\text { Glasgow Come Scale at } \\
\text { discharge }\end{array}$ & Median & Median & \\
\hline Level of consciousness & 15 & 14 & $0.211^{\S}$ \\
\hline $\mathrm{mRS}$ at discharge & $n(\%)$ & $\mathrm{n}(\%)$ & \\
\hline $2-3$ & $12(60)$ & $11(58)$ & - \\
\hline $4-5$ & $6(30)$ & $8(40)$ & $0.515^{\#}$ \\
\hline Mortality & $\mathrm{n}(\%)$ & $\mathrm{n}(\%)$ & \\
\hline In hospital death & $2(10)$ & 00 & $0.4877^{\#}$ \\
\hline \multicolumn{4}{|c|}{$\begin{array}{l}\text { n: number; SD: standard deviation; CSF: cerebro-spinal fluid; EVD: external ventricular drain; CVS: } \\
\text { cerebral vasospasm; mRS: modified Rankin scale; } § \text { Mann-Whitney-U test for continuous parameters; } \\
\text { \#Fisher's exact chi-squared test for categorical parameters; }{ }^{+} \text {t-test for numerical variables; statistical } \\
\text { significance was at alpha level of } P=.05 ;\end{array}$} \\
\hline
\end{tabular}


Table 3

Univariable covariate logistic regression analysis for early permanent CSF diversion ( $<14$ days), with the primary endpoint EVD-associated infection (EVDAI) and the secondary endpoints delayed cerebral vasospasms (DCVS), delayed cortical infarcts (DCl) and poor outcome at discharge (mRS 4-6). The univariable binary logistic regression model includes the following covariates: age $\geq 60$ years (reference level: age < 60 years), female sex (reference level: male sex), anterior circulation (reference level: posterior circulation), poor WFNS-score (reference level: WFNS-score 1-3), high BNI-score (reference level: BNI score 1-3), endovascular treatment (reference level: surgical clipping), EVD duration (continuous variable), presence of delayed cerebral vasospasm or delayed cortical infarction (reference level: absence), presence of a focal neurological and cranial nerve deficit at discharge (reference level: absence), hospitalization time (continuous variable) and early permanent CSF diversion (reference level: late permanent CSF diversion after 14 days). Statistical significance was set at alpha level of $p=.05$. Significance is indicated as follows: * $(p \leq .05), * \star(p \leq .01)$.

\section{Univariable Analysis}

\begin{tabular}{|lllll|}
\hline Early permanent CSF diversion ( $\leq 14$ days) & OR & $95 \%-C l$ & P-value \\
\hline Age $\geq 60$ years & & Lower & Upper & \\
\hline Female sex & 0.90 & 0.26 & 3.16 & 0.869 \\
\hline Poor WFNS (4-5) on admission & 1.67 & 0.47 & 5.93 & 0.430 \\
\hline High BNI score (4-5) on admission & 0.36 & 0.09 & 1.37 & 0.134 \\
\hline Intraventricular hemorrhage & 1.38 & 0.34 & 5.62 & 0.684 \\
\hline EVD in situ (per day) & 0.36 & 0.06 & 2.01 & 0.252 \\
\hline Delayed cerebral vasospasms & 0.73 & 0.58 & 0.91 & $0.006 * \star$ \\
\hline Delayed cortical infarction & 0.60 & 0.17 & 2.14 & 0.430 \\
\hline Poor mRS (4-6) at discharge & 0.34 & 0.08 & 1.43 & 0.142 \\
\hline Focal neurological deficit at discharge & 0.92 & 0.26 & 3.29 & 0.894 \\
\hline Cranial nerve deficit at discharge & 1.07 & 0.22 & 5.13 & 0.931 \\
\hline Hospitalization time (per day) & 1.06 & 0.13 & 8.47 & 0.954 \\
\hline EVD-associated infection & 0.93 & 0.84 & 1.02 & 0.117 \\
\hline
\end{tabular}

EVD: External ventricular drain, CSF: cerebro-spinal fluid; BNI: Barrow Neurological Institute, WFNS: World Federation of Neurosurgical Societies, mRS: modified Rankin Scale 


\section{Univariable Analysis}

\begin{tabular}{|c|c|c|c|c|}
\hline EVD-associated infection & OR & $95 \%-\mathrm{Cl}$ & & p-value \\
\hline & & Lower & Upper & \\
\hline Age $\geq 60$ years & 0.49 & 0.10 & 2.44 & 0.387 \\
\hline Female Sex & 0.82 & 0.17 & 3.90 & 0.807 \\
\hline Poor WFNS (4-5) on admission & 2.17 & 0.38 & 12.49 & 0.387 \\
\hline High BNI-Score (4-5) on admission & 0.29 & 0.06 & 1.48 & 0.136 \\
\hline Intraventricular hemorrhage & 1.68 & 0.17 & 16.4 & 0.655 \\
\hline EVD in situ (per day) & 1.14 & 1.00 & 1.30 & $0.010^{*}$ \\
\hline Poor mRS (4-6) at discharge & 0.40 & 0.07 & 2.33 & 0.890 \\
\hline Hospitalisation time (per day) & 1.01 & 0.91 & 1.12 & 0.120 \\
\hline Early permanent CSF-diversion ( $\leq 14$ days) & 0.09 & 0.01 & 0.83 & $0.030^{\star}$ \\
\hline Delayed Cerebral Vasospasm & OR & $95 \%-\mathrm{Cl}$ & & $\mathrm{p}$-value \\
\hline & & Lower & Upper & \\
\hline Age $\geq 60$ years & 0.60 & 0.17 & 2.14 & 0.430 \\
\hline Female sex & 0.49 & 0.14 & 1.77 & 0.278 \\
\hline Anterior circulation aneurysm & 1.25 & 0.18 & 8.49 & 0.819 \\
\hline Poor WFNS (4-5) on admission & 2.36 & 0.62 & 9.03 & 0.209 \\
\hline High BNI score (4-5) on admission & 0.37 & 0.09 & 1.57 & 0.177 \\
\hline Endovascular treatment & 5.00 & 0.52 & 47.73 & 0.162 \\
\hline EVD in situ (per day) & 1.04 & 0.94 & 1.14 & 0.424 \\
\hline Delayed cortical infarction & 11.87 & 2.11 & 66.87 & $0.005^{\star \star}$ \\
\hline Poor mRS (4-6) at discharge & 5.03 & 1.26 & 20.00 & $0.022^{\star}$ \\
\hline Focal neurological deficit at discharge & 5.70 & 0.97 & 33.60 & 0.054 \\
\hline Cranial nerve deficit at discharge & 0.40 & 0.04 & 4.26 & 0.448 \\
\hline
\end{tabular}

EVD: External ventricular drain, CSF: cerebro-spinal fluid; BNI: Barrow Neurological Institute, WFNS: World Federation of Neurosurgical Societies, mRS: modified Rankin Scale 


\section{Univariable Analysis}

\begin{tabular}{|c|c|c|c|c|}
\hline Hospitalization time (per day) & 1.13 & 1.01 & 1.25 & $0.030 *$ \\
\hline EVD-associated infection & 0.64 & 0.13 & 3.16 & 0.583 \\
\hline Early permanent CSF diversion ( $\leq 14$ days) & 0.60 & 0.17 & 2.14 & 0.430 \\
\hline \multirow[t]{2}{*}{ Delayed Cerebral Infarction } & OR & $95 \%-\mathrm{Cl}$ & & $\mathrm{p}$-value \\
\hline & & Lower & Upper & \\
\hline Age $\geq 60$ years & 1.51 & 0.38 & 5.96 & 0.558 \\
\hline Female sex & 1.30 & 0.33 & 5.13 & 0.708 \\
\hline Anterior circulation aneurysm & 0.56 & 0.08 & 3.94 & 0.562 \\
\hline Poor WFNS (4-5) on admission & 1.37 & 0.33 & 5.72 & 0.661 \\
\hline High BNI score (4-5) on admission & 0.70 & 0.16 & 3.07 & 0.636 \\
\hline EVD in situ (per day) & 1.08 & 0.97 & 1.20 & 0.140 \\
\hline Delayed cerebral vasospasms & 11.87 & 2.11 & 66.87 & $0.005^{\star \star}$ \\
\hline Poor mRS (4-6) at discharge & 2.80 & 0.69 & 11.34 & 0.149 \\
\hline Focal neurological deficit at discharge & 0.88 & 0.15 & 5.27 & 0.884 \\
\hline Cranial nerve deficit at discharge & 0.89 & 0.08 & 9.69 & 0.923 \\
\hline Hospitalization time (per day) & 0.99 & 0.90 & 1.09 & 0.796 \\
\hline EVD-associated infection & 1.47 & 0.29 & 7.47 & 0.645 \\
\hline Early permanent CSF diversion ( $\leq 14$ days) & 0.34 & 0.08 & 1.43 & 0.142 \\
\hline \multirow[t]{2}{*}{ Poor mRS (4-6) at discharge } & OR & $95 \%-\mathrm{Cl}$ & & $\mathrm{p}$-value \\
\hline & & Lower & Upper & \\
\hline Age $\geq 60$ years & 0.60 & 0.17 & 2.17 & 0.434 \\
\hline Female sex & 0.77 & 0.21 & 2.77 & 0.688 \\
\hline Anterior circulation aneurysm & 2.95 & 0.30 & 29.32 & 0.356 \\
\hline Poor WFNS (4-5) on admission & 4.73 & 1.06 & 21.15 & $0.042^{*}$ \\
\hline High BNI score (4-5) on admission & 1.31 & 0.31 & 5.53 & 0.711 \\
\hline
\end{tabular}

EVD: External ventricular drain, CSF: cerebro-spinal fluid; BNI: Barrow Neurological Institute, WFNS: World Federation of Neurosurgical Societies, mRS: modified Rankin Scale 


\section{Univariable Analysis}

\begin{tabular}{|c|c|c|c|c|}
\hline Endovascular Treatment & 3.89 & 0.41 & 37.18 & 0.238 \\
\hline EVD in situ (per day) & 1.00 & 0.91 & 1.10 & 0.992 \\
\hline Delayed cerebral vasospasms & 5.03 & 1.26 & 20.00 & $0.022^{*}$ \\
\hline Delayed cortical infarction & 2.80 & 0.69 & 11.34 & 0.149 \\
\hline Hospitalization time (per day) & 0.95 & 0.87 & 1.05 & 0.328 \\
\hline EVD-associated infection & 0.40 & 0.07 & 2.33 & 0.311 \\
\hline Early permanent CSF-diversion ( $\leq 14$ days) & 0.92 & 0.26 & 3.29 & 0.894 \\
\hline
\end{tabular}

EVD-associated infections: The prevalence of EVDAI was 8 out of 39 (20.5\%). The rate of colonization of the EVD catheter was $7 / 20$ (35\%) in the early group and 7/19 (37\%) in the late group, but the EVDAI rate was much lower in the early group 1/20 (5\%) than in the late group 7/19 (35\%). The prevalence per 100 persons, the incidence rate per EVD day in situ, and the cumulative incidence were approximately 5 times higher in the late group than in the early group (Table 2). In multivariable logistic regression analysis, EVDAI were less likely to occur with shorter EVD duration and early permanent CSF diversion (Table 3, Fig. $2 \mathrm{C}$ and $\mathrm{D}$ ).

Permanent CSF diversion: Mean time to implantation of a permanent CSF diversion device was $12( \pm 2)$ days in the early and $21( \pm 6)$ days in the late group. Selected relevant CSF-values on the day of operation did not show any differences between the groups. The overall occurrence of permanent CSF diversion related complications within the first month was 5 out of 39 (12.8\%), consisting of obstruction of the CSF diversion device, infectious complications, or malposition of the CSF diversion device tip. There was no statistically significant difference between the groups (Table 2).

Symptomatic cerebral vasospasm: The rate of symptomatic DCVS in the present cohort was 18/39 (46.1\%). The mean interval from the ictus to the onset of symptomatic DCVS was $12( \pm 6)$ days in the early group and $8.5( \pm 4)$ days in the late group. In the early group, three patients developed DCVS before and five patients after the CSF diversion procedure; in the late group, all cases of DCVS arose before the procedure (Table 2). Three patients in the early group developed symptomatic DCVS within 48 hours of the CSF diversion procedure (Table 4). Multivariable logistic regression analysis did not reveal any association between DCVS and the timing of the permanent CSF diversion procedure (Table 3 and Fig. 3A). 
Table 4

Descriptive table of patients suffering from delayed cerebral vasospasms (DCVS) within 48 hours after permanent CSF diversion procedure

\section{CVS onset within 48 hours after permanent CSF diversion procedure $\quad$ Case 1 Case 2 Case 3}

Permanent CSF diversion

Implantation after ictus (days)

10

13

10

Baseline characteristics

Age (years)

56

49

56

GCS at admission

53

WFNS on admission

5

3

12

Fisher Grade (I-IV)

5

4

Fisher Grade (IIV)

IV

IV

IV

BNI score

4

$\mathrm{BNI}$ in $\mathrm{mm}$

11

Acute Hydrocephalus (CT $<72$ h)

Intraventricular hemorrhage

$\mathrm{mRS}$ at discharge

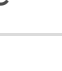

EVD

Total days with EVD

$\begin{array}{lll}10 & 11 & 10 \\ \text { Yes } & \text { Yes } & \text { Yes }\end{array}$

Unsuccessfully weaning

Yes

Yes

2

5

Aneurysm treatment

Surgical clip ligation

Endovascular treatment

$\begin{array}{lll}\text { No } & \text { Yes } & \text { No } \\ \text { Yes } & \text { No } & \text { Yes }\end{array}$

Delayed cerebral vasospasms

Confirmed by CTA, TCD and DSA

$\begin{array}{lll}\text { Yes } & \text { Yes } & \text { Yes } \\ 12 & 15 & 5 \\ 2 & 2 & 1\end{array}$

CSF: cerebro-spinal fluid; GCS: Glasgow Coma Scale; WNFS: World Federation of Neurological Societies; BNI: Barrow Neurological Institute; mRS: modified Ranking Scale, EDV: external ventricle drain; CTA: computed tomography angiography; TCD: transcranial Doppler; DSA: digital subtraction angiography; CT: computed tomography 


\section{CVS onset within 48 hours after permanent CSF diversion procedure $\quad$ Case 1 Case 2 Case 3}

\begin{tabular}{|c|c|c|c|}
\hline Treatment by balloon dilation & No & No & Yes \\
\hline Treatment by chemical dilation & Yes & No & Yes \\
\hline \multicolumn{4}{|l|}{ Delayed cortical infarct } \\
\hline Confirmed by CT & No & No & Yes \\
\hline \multicolumn{4}{|l|}{ Mortality } \\
\hline In hospital death & No & No & Yes \\
\hline
\end{tabular}

Delayed cerebral infarction: Infarcts were detected by CT in 14/39 (35.9\%) patients by the time of discharge (Table 2). Multivariable logistic regression analysis revealed no association between $\mathrm{DCl}$ and the timing of the permanent CSF diversion procedure (Table 3 and Fig. 3B).

Poor neurological outcome and in-hospital death: The percentage of patients with a low mRS on discharge did not differ between the two groups (Table 2 ). The overall mortality was $2 / 39$ (5\%), with both deaths occurring in the early permanent CSF diversion group. Both of these patients suffered from extensive DCl due to DCVS; in one of them, DCV

$S$ arose within 48 hours of the CSF diversion procedure (Table 4). In multivariable logistic regression analysis, the timing of the CSF diversion procedure had no effect on outcome (Table 3 and Fig. $3 \mathrm{C}$ ).

\section{Discussion}

Acute hydrocephalus remains an important adverse sequela of aSAH [1, 2]. Temporary CSF diversion with an EVD is generally considered the main management approach in the acute phase of the disease in patients with impaired consciousness [10]. Over time, this mode of treatment carries a high risk of nosocomial infection. Induced immunodepression in the subset of aSAH patients has been determined to predispose for bacterial infections and hence significantly promoting a poor outcome [4-6, 37]. Several recent studies have addressed the relevant risk factors and have established tools to predict the need for a permanent CSF diversion after aSAH $[3,10,16,17]$. The initial EVD placement itself has been found to be correlated with permanent CSF diversion aSAH $[3,10]$. Increasing evidence also suggests that EVDAl is an independent risk factor for permanent CSF diversion in aSAH. Adams et al. [10] found that aSAH with EVDAl carried the highest risk (61\%), with only $50 \%$ of patients not having CSF diversion at 1 year. According to a recent meta-analysis, patients with aSAH who sustained in-hospital complications were 5 times more likely to undergo permanent CSF diversion than those who did not [3]; among all in-hospital, complications, EVDAI conferred the highest risk for permanent CSF diversion [3]. The overall infection rate 
in the present study was $20.5 \%$, the majority in patients who received a permanent CSF diversion more than two weeks after the ictus (Table 2, Fig. 2A and B). The mechanisms behind the transition of hydrocephalus from an acute to a chronic condition have yet to be fully understood. Inflammation induced by EVDAI can lead to CSF circulation disturbances through ependymal cell dysfunction, CSF barrier cell inflammation with increased CSF secretion, and infectious debris blocking CSF outflow [8-10]. Interestingly, the present study demonstrated a higher ratio of catheter colonization to EVDAl in the early group than in the late group (7/1 out of 20 patients vs. 7/7 out of 19 patients; Table 2). Accordingly, EVDAI were found to be associated with longer EVD duration and later permanent CSF diversion (Table 3, Fig. $2 \mathrm{C}$ and $\mathrm{D}$ ). From a pathophysiological perspective, longer EVD duration promotes progressive catheter colonization from growing skin flora contaminants over time that might induce inflammatory processes. Pathogens at the skin site and CSF leakage were found to be independent predictors of EVDAI $[9,38]$. As previously discussed, a predisposition for bacterial infection induced by immunodepression might accentuate this risk specifically in the subset of aSAH patients [4-6,37]. However, the exact interplay between these factors has yet to be elucidated, and a rather complex interaction between dynamic systems seems more likely than a simple linear causation [11]. Nevertheless, EVDAI is partially preventable and constitutes one of the factors that can be influenced directly. Hence, efforts to avoid it through protection of the EVD entry site, strict EVD-protocols, the use of silver-impregnated catheters, and minimizing the duration of EVD are crucially important $[9,10,27,39-41]$.

The present study revealed higher levels of consciousness in patients who received early permanent CSF diversion (Table 1). A potential explanation might be the influence of the aSAH grade on the surgeon's decision despite the initial radiological and laboratory blood clot burden being similarly distributed between the groups. In general, the timing of EVD weaning and of conversion to a permanent CSF diversion in aSAH patients is probably not optimal today, although the available literature offers only sparse evidence on the matter. Klopfenstein et al. [42] previously reported no advantage from rapid weaning compared to gradual, multi-step weaning in lessening the need for permanent CSF diversion. Kang et al. [27] demonstrated that earlier EVD weaning and permanent CSF diversion effectively treated $\mathrm{SAH}$-induced hydrocephalus in poor-grade aSAH patients. The laboratory blood clot burden, reflected by the count of red blood cells (RBC) and protein in CSF, is used by many neurosurgeons to determine the right time-point for a permanent CSF diversion procedure after aSAH. However, the evidence remains somewhat equivocal. A level of $2000 \mathrm{RBCs} / \mu \mathrm{L}$ has been reported as a safe margin to perform such a procedure $[25,26]$. Brydon et al. found a positive correlation between a high RBC count in the CSF and CSF diversion device obstruction, while the amount of CSF protein, on the other hand, did not demonstrate such a correlation [26]. In contrast, Rammos et al. [25] reported of 80 patients who had undergone conversion of an EVD to a permanent CSF diversion after aSAH-induced hydrocephalus, and did not confirm the said correlation. The present study revealed elevated mean values of perioperative CSF proteins, RBCs, and leucocytes in both groups, with higher mean RBC-counts in the early group (Table 2). The overall rate of permanent CSF diversion device complications was 5 out of $39(12.2 \%)$. However, only two cases of permanent CSF diversion device tip obstruction were encountered (2 out of 
$39,5.2 \%$ ), of which one occurred in each group (Table 2 ). The findings of the present study are in accordance with the conflicting findings of the previously described studies $[25,26]$.

Based on the current results, early EVD-weaning and conversion to a permanent CSF diversion results in shorter EVD duration, and might be justified in more severe SAH with intraventricular hemorrhage (Table 2). Besides reduced infectious complications, shorter EVD duration might lead to earlier mobilization of the patient with reduced pulmonary and systemic complications, and hence reduced hospitalization time and cost-reduction [27, 43]. However, concerns might remain that such a surgical procedure might cause hemodynamic disturbances during a period of impaired cerebrovascular autoregulation, and thereby increase the risk of cerebral hypoperfusion [20-22]. A recent study found that patients receiving surgical clip ligation had a higher cardiac output with early postoperative hypovolemia when compared to endovascular treatment $[23,24]$. The surgical treatment resulted in a poorer cardiac preload responsiveness to volume, requiring more intravenous volume to maintain normovolemia in the early postoperative period $[23,24]$. This might be of special importance in poorer grade aSAH patients where hemodynamic insufficiency related to hypovolemia or low cardiac output is present [24]. Data on CSF diversion procedures during the first two weeks after aSAH are lacking [20-22].

The present study could confirm a positive correlation between DCVS and the presence of DCl at discharge in the univariable analysis. Multivariable and logistic regression analysis, however, revealed no correlation between the timing of the permanent CSF diversion procedure and the occurrence of DCVS and $\mathrm{DCl}$. This is further supported by the fact that only 3 out of 8 patients with DCVS in patients receiving early permanent CSF-diversion were diagnosed within 48 hours after surgery (Table 4). This may be because $\mathrm{DCl}$ is already largely predicted by other, known factors, including initial blood clot burden and DCVS (Table 3 and Fig. 3A and B) [44].

The present study did not reveal any association between the timing of CSF diversion and clinical outcome or in-hospital mortality (Table 3 and Fig. 3C). Any influence of the timing of CSF diversion, even if present, may have been rendered undetectable by the much larger influence of the known, strong predictors of poor outcome and in-hospital death [45].

\section{Strengths And Limitations}

This study is subject to all the inherent limitations arising from retrospective data collection. Because of the small sample size, no definitive general recommendation can be made for early permanent CSF diversion in aSAH patients. The timing of EVD weaning and permanent CSF diversion were up to the treating surgeon in all cases, and selection bias may have resulted. Yet, selection bias is inevitable in daily clinical routine. The strengths of this study include the homogenous distribution of the two compared groups, which were treated in a single center with uniform surgical standards and materials, and a clear definition of the primary (EVDAI and DCVS) and secondary (DCI) outcomes. For the sake of data consistency, the authors assessed outcomes only at the time of discharge from the hospital. 


\section{Conclusion}

Permanent CSF diversion procedures performed within the first two weeks of aSAH are associated with a shorter temporary CSF diversion period and a lower prevalence of EVDAl. Poor mRS resulting from DCVS and $\mathrm{DCl}$ did not correlate with the timing of the intervention. Early permanent CSF diversion might be a way to prevent infection in good-grade aSAH patients. Further retrospective or prospective studies with larger cohorts are warranted to confirm these results.

\section{List Of Abbreviations}

aSAH aneurysmal subarachnoid hemorrhage

BNI Barrow Neurological Institute Scale

CSF Cerebrospinal fluid

DCVS Delayed Cerebral Vasospasm

DCI Delayed Cortical Ischemia

EKNZ Ethik-Kommission Nordwest Schweiz

EVD External ventricular drain

EVDAI External ventricular drain associated infections

GCS Glasgow Coma Scale

IRB Institutional Review Board

IVH Inraventricular hemorrhage

mRS Modified Rankin Scale

SOS Swiss Study of Subarachnoid Hemorrhage Registry

WFNS World Federation Neurological-Surgeon Scale

\section{Declarations}

\section{Ethical approval and consent to participate}

Institutional Review Board (IRB) and local ethics committee (EKNZ, Basel, Switzerland) approval was obtained and accredited (Project-ID 2018-02129). The ethics committee waived the requirement for written informed consent (justification: disproportionality) for patients recruited prior to 2014, when the 
new Swiss Human Research Act went in force. As of 2014, written informed consent was obtained from all participants.

\section{Consent for publication}

Not applicable.

\section{Availability of data and materials}

The datasets used and/or analyzed during the current study are available from the corresponding author on reasonable request.

\section{Competing interests}

The authors declare that they have no competing interests.

\section{Funding}

This study was funded by the Martin Allgöwer Foundation, the Departments of Surgery and Neurosurgery of the University Hospital Basel (Basel, Switzerland), and the Gottfried and Julia Bangerter-Rhyner Foundation (Bern, Switzerland). The University Hospital of Basel (Basel, Switzerland) and the University of Malaya Specialist Centre (Kuala Lumpur, Malaysia) supported this study.

\section{Authors' contributions}

DMC and MR: Design and concept of the study, collection of data, and drafting of the manuscript. MD: Collection of data. SA and MR: Statistical analysis, result report, creation of tables and figures. ET: English proofread of the manuscript. All authors: revision of final manuscript. MR: Final responsibility and submission of the manuscript.

\section{Acknowledgments}

The authors thank Selina Ackermann, University Hospital of Basel, for proofreading the manuscript and submission support. M.R. thanks Prof. Vicknes Waran, Neurosurgery Department, University of Malaya Specialist Center, for mentoring and support during the clinical research fellowship 2018-2019.

\section{References}

1. Hop JW, Rinkel GJ, Algra A, van Gijn J: Case-fatality rates and functional outcome after subarachnoid hemorrhage: a systematic review. Stroke 1997, 28(3):660-664.

2. Graff-Radford NR, Torner J, Adams HP, Jr., Kassell NF: Factors associated with hydrocephalus after subarachnoid hemorrhage. A report of the Cooperative Aneurysm Study. Arch Neuro/ 1989, 46(7):744-752. 
3. Wilson CD, Safavi-Abbasi S, Sun H, Kalani MY, Zhao YD, Levitt MR, Hanel RA, Sauvageau E, Mapstone TB, Albuquerque FC et al: Meta-analysis and systematic review of risk factors for shunt dependency after aneurysmal subarachnoid hemorrhage. J Neurosurg 2017, 126(2):586-595.

4. Ramanan M, Lipman J, Shorr A, Shankar A: A meta-analysis of ventriculostomy-associated cerebrospinal fluid infections. BMC Infect Dis 2015, 15:3.

5. Lozier AP, Sciacca RR, Romagnoli MF, Connolly ES: Ventriculostomy-related infections: a critical review of the literature. Neurosurgery 2008, 62 Suppl 2:688-700.

6. Hoefnagel D, Dammers R, Ter Laak-Poort MP, Avezaat CJ: Risk factors for infections related to external ventricular drainage. Acta Neurochir (Wien) 2008, 150(3):209-214; discussion 214.

7. Champey J, Mourey C, Francony G, Pavese P, Gay E, Gergele L, Manet R, Velly L, Bruder N, Payen JF: Strategies to reduce external ventricular drain-related infections: a multicenter retrospective study. $J$ Neurosurg 2018:1-6.

8. Simard PF, Tosun C, Melnichenko L, Ivanova S, Gerzanich V, Simard JM: Inflammation of the choroid plexus and ependymal layer of the ventricle following intraventricular hemorrhage. Trans/ Stroke Res 2011, 2(2):227-231.

9. Roethlisberger M, Moffa G, Fisch U, Wiggli B, Schoen S, Kelly C, Leu S, Croci D, Zumofen DW, Cueni N et al: Effectiveness of a Chlorhexidine Dressing on Silver-coated External Ventricular Drainassociated Colonization and Infection: A Prospective Single-blinded Randomized Controlled Clinical Trial. Clin Infect Dis 2018, 67(12):1868-1877.

10. Adams H, Ban VS, Leinonen V, Aoun SG, Huttunen J, Saavalainen T, Lindgren A, Frosen J, Fraunberg M, Koivisto T et al: Risk of Shunting After Aneurysmal Subarachnoid Hemorrhage: A Collaborative Study and Initiation of a Consortium. Stroke 2016, 47(10):2488-2496.

11. Foreman PM, Chua M, Harrigan MR, Fisher WS, 3rd, Vyas NA, Lipsky RH, Walters BC, Tubbs RS, Shoja $\mathrm{MM}$, Griessenauer $\mathrm{CJ}$ : Association of nosocomial infections with delayed cerebral ischemia in aneurysmal subarachnoid hemorrhage. J Neurosurg 2016, 125(6):1383-1389.

12. Kasius KM, Frijns CJ, Algra A, Rinkel GJ: Association of platelet and leukocyte counts with delayed cerebral ischemia in aneurysmal subarachnoid hemorrhage. Cerebrovascular diseases (Basel, Switzerland) 2010, 29(6):576-583.

13. McMahon CJ, Hopkins S, Vail A, King AT, Smith D, Illingworth KJ, Clark S, Rothwell NJ, Tyrrell PJ: Inflammation as a predictor for delayed cerebral ischemia after aneurysmal subarachnoid haemorrhage. J Neurointerv Surg 2013, 5(6):512-517.

14. Muroi C, Hugelshofer M, Seule M, Tastan I, Fujioka M, Mishima K, Keller E: Correlation among systemic inflammatory parameter, occurrence of delayed neurological deficits, and outcome after aneurysmal subarachnoid hemorrhage. Neurosurgery 2013, 72(3):367-375; discussion 375.

15. Lewis A, Taylor Kimberly W: Prediction of ventriculoperitoneal shunt placement based on type of failure during external ventricular drain wean. Clin Neurol Neurosurg 2014, 125:109-113.

16. Jabbarli R, Bohrer AM, Pierscianek D, Muller D, Wrede KH, Dammann P, El Hindy N, Ozkan N, Sure U, Muller O: The CHESS score: a simple tool for early prediction of shunt dependency after aneurysmal 
subarachnoid hemorrhage. European journal of neurology : the official journal of the European Federation of Neurological Societies 2016, 23(5):912-918.

17. Diesing D, Wolf S, Sommerfeld J, Sarrafzadeh A, Vajkoczy P, Dengler NF: A novel score to predict shunt dependency after aneurysmal subarachnoid hemorrhage. J Neurosurg 2018, 128(5):12731279 .

18. Kassell NF, Sasaki T, Colohan AR, Nazar G: Cerebral vasospasm following aneurysmal subarachnoid hemorrhage. Stroke 1985, 16(4):562-572.

19. Kassell NF, Torner JC, Haley EC, Jane JA, Adams HP, Kongable GL: The International Cooperative Study on the Timing of Aneurysm Surgery. Part 1: Overall management results. J Neurosurg 1990, 73(1):18-36.

20. Gritti P, Lorini FL, Lanterna LA, Bilotta F: Periprocedural management of patients with subarachnoid hemorrhage. Curr Opin Anaesthesio/ 2018, 31(5):511-519.

21. Keyrouz SG, Diringer MN: Clinical review: Prevention and therapy of vasospasm in subarachnoid hemorrhage. Crit Care 2007, 11(4):220.

22. Karic T, Roe C, Nordenmark TH, Becker F, Sorteberg W, Sorteberg A: Effect of early mobilization and rehabilitation on complications in aneurysmal subarachnoid hemorrhage. J Neurosurg 2017, 126(2):518-526.

23. Mutoh T, Kazumata K, Yokoyama Y, Ishikawa T, Taki Y, Terasaka S, Houkin K: Comparison of postoperative volume status and hemodynamics between surgical clipping and endovascular coiling in patients after subarachnoid hemorrhage. J Neurosurg Anesthesiol 2015, 27(1):7-15.

24. Gruenbaum SE, Bilotta F: Postoperative ICU management of patients after subarachnoid hemorrhage. Curr Opin Anaesthesiol 2014, 27(5):489-493.

25. Rammos S, Klopfenstein J, Augspurger L, Wang H, Wagenbach A, Poston J, Lanzino G: Conversion of external ventricular drains to ventriculoperitoneal shunts after aneurysmal subarachnoid hemorrhage: effects of site and protein/red blood cell counts on shunt infection and malfunction. $J$ Neurosurg 2008, 109(6):1001-1004.

26. Brydon HL, Bayston R, Hayward R, Harkness W: The effect of protein and blood cells on the flowpressure characteristics of shunts. Neurosurgery 1996, 38(3):498-504; discussion 505.

27. Kang DH, Park J, Park SH, Kim YS, Hwang SK, Hamm IS: Early ventriculoperitoneal shunt placement after severe aneurysmal subarachnoid hemorrhage: role of intraventricular hemorrhage and shunt function. Neurosurgery 2010, 66(5):904-908; discussion 908-909.

28. Shigematsu H, Sorimachi T, Osada T, Aoki R, Srivatanakul K, Oda S, Matsumae M: Predictors of early vs. late permanent shunt insertion after aneurysmal subarachnoid hemorrhage. Neurological research 2016, 38(7):600-605.

29. Teasdale G, Jennett B: Assessment of coma and impaired consciousness. A practical scale. Lancet 1974, 2(7872):81-84.

30. Teasdale GM, Drake CG, Hunt W, Kassell N, Sano K, Pertuiset B, De Villiers JC: A universal subarachnoid hemorrhage scale: report of a committee of the World Federation of Neurosurgical 
Societies. J Neurol Neurosurg Psychiatry 1988, 51(11):1457.

31. Fisher CM, Kistler JP, Davis JM: Relation of cerebral vasospasm to subarachnoid hemorrhage visualized by computerized tomographic scanning. Neurosurgery 1980, 6(1):1-9.

32. Wilson DA, Nakaji P, Abla AA, Uschold TD, Fusco DJ, Oppenlander ME, Albuquerque FC, McDougall CG, Zabramski JM, Spetzler RF: A simple and quantitative method to predict symptomatic vasospasm after subarachnoid hemorrhage based on computed tomography: beyond the Fisher scale. Neurosurgery 2012, 71(4):869-875.

33. Farrell B, Godwin J, Richards S, Warlow C: The United Kingdom transient ischaemic attack (UK-TIA) aspirin trial: final results. J Neurol Neurosurg Psychiatry 1991, 54(12):1044-1054.

34. Garner JS, Jarvis WR, Emori TG, Horan TC, Hughes JM: CDC definitions for nosocomial infections, 1988. Am J Infect Control 1988, 16(3):128-140.

35. Sobey CG, Faraci FM: Subarachnoid haemorrhage: what happens to the cerebral arteries? Clin Exp Pharmacol Physiol 1998, 25(11):867-876.

36. Vergouwen MD, Vermeulen M, van Gijn J, Rinkel GJ, Wijdicks EF, Muizelaar JP, Mendelow AD, Juvela $\mathrm{S}$, Yonas $\mathrm{H}$, Terbrugge $\mathrm{KG}$ et al: Definition of delayed cerebral ischemia after aneurysmal subarachnoid hemorrhage as an outcome event in clinical trials and observational studies: proposal of a multidisciplinary research group. Stroke 2010, 41(10):2391-2395.

37. Sarrafzadeh A, Schlenk F, Meisel A, Dreier J, Vajkoczy P, Meisel C: Immunodepression after aneurysmal subarachnoid hemorrhage. Stroke 2011, 42(1):53-58.

38. Mounier R, Lobo D, Cook F, Martin M, Attias A, Ait-Mamar B, Gabriel I, Bekaert O, Bardon J, Nebbad B et al: From the Skin to the Brain: Pathophysiology of Colonization and Infection of External Ventricular Drain, a Prospective Observational Study. PloS one 2015, 10(11):e0142320.

39. Keong NC, Bulters DO, Richards HK, Farrington M, Sparrow OC, Pickard JD, Hutchinson PJ, Kirkpatrick PJ: The SILVER (Silver Impregnated Line Versus EVD Randomized trial): a double-blind, prospective, randomized, controlled trial of an intervention to reduce the rate of external ventricular drain infection. Neurosurgery 2012, 71(2):394-403; discussion 403-394.

40. Flint AC, Rao VA, Renda NC, Faigeles BS, Lasman TE, Sheridan W: A simple protocol to prevent external ventricular drain infections. Neurosurgery 2013, 72(6):993-999; discussion 999.

41. Flint AC, Toossi S, Chan SL, Rao VA, Sheridan W: A Simple Infection Control Protocol Durably Reduces External Ventricular Drain Infections to Near-Zero Levels. World neurosurgery 2017, 99:518523.

42. Klopfenstein JD, Kim LJ, Feiz-Erfan I, Hott JS, Goslar P, Zabramski JM, Spetzler RF: Comparison of rapid and gradual weaning from external ventricular drainage in patients with aneurysmal subarachnoid hemorrhage: a prospective randomized trial. J Neurosurg 2004, 100(2):225-229.

43. Hasan D, Vermeulen M, Wijdicks EF, Hijdra A, van Gijn J: Management problems in acute hydrocephalus after subarachnoid hemorrhage. Stroke 1989, 20(6):747-753.

44. Neidert MC, Maldaner N, Stienen MN, Roethlisberger M, Zumofen DW, D'Alonzo D, Marbacher S, Maduri R, Hostettler IC, Schatlo B et al: The Barrow Neurological Institute Grading Scale as a 
Predictor for Delayed Cerebral Ischemia and Outcome After Aneurysmal Subarachnoid Hemorrhage: Data From a Nationwide Patient Registry (Swiss SOS). Neurosurgery 2018, 83(6):1286-1293.

45. Stienen MN, Germans M, Burkhardt JK, Neidert MC, Fung C, Bervini D, Zumofen D, Rothlisberger M, Marbacher S, Maduri R et al: Predictors of In-Hospital Death After Aneurysmal Subarachnoid Hemorrhage: Analysis of a Nationwide Database (Swiss SOS [Swiss Study on Aneurysmal Subarachnoid Hemorrhage]). Stroke 2018, 49(2):333-340.

\section{Figures}




\section{Inclusion Criteria}

- Aneurysmal subarachnoid hemorrhage on admission

- Temporary extra-corporal CSF diversion with external ventricular drain (EVD)

- Permanent CSF diversion procedure during the same hospitalization

Excluded $(n=235)$

- $\quad$ Not meeting inclusion criteria
Early permanent CSF diversion $\leq 14$ days after aSAH ( $n=20)$
Primary Endpoint Assessment $(n=20)$

EVD-associated Infection

Secondary Endpoint Assessment ( $n=20)$

Symptomatic cerebral vasospasm

Delayed cerebral ischemia

Modified Rankin Scale at discharge

Temporary CSF diversion related data Permanent CSF diversion related data Cerebral vasospasm related variables Duration of hospitalization In hospital death
Eligible for analysis $(n=39)$
Late permanent CSF diversion $>15$ days after aSAH $(n=19)$

\section{Figure 1}

\section{Patient inclusion profile}



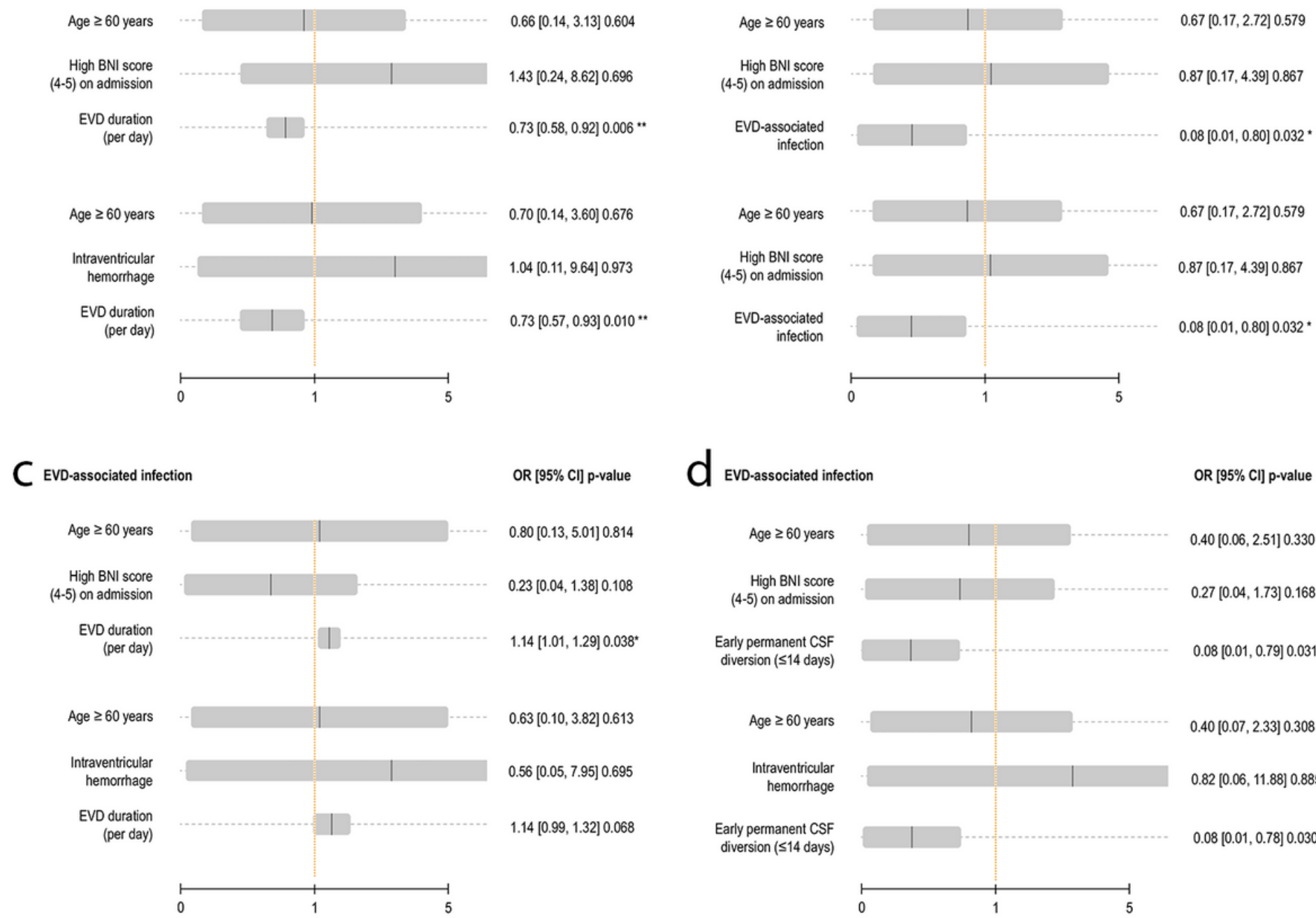

EVD-associated infection

OR [95\% Cl] p-value

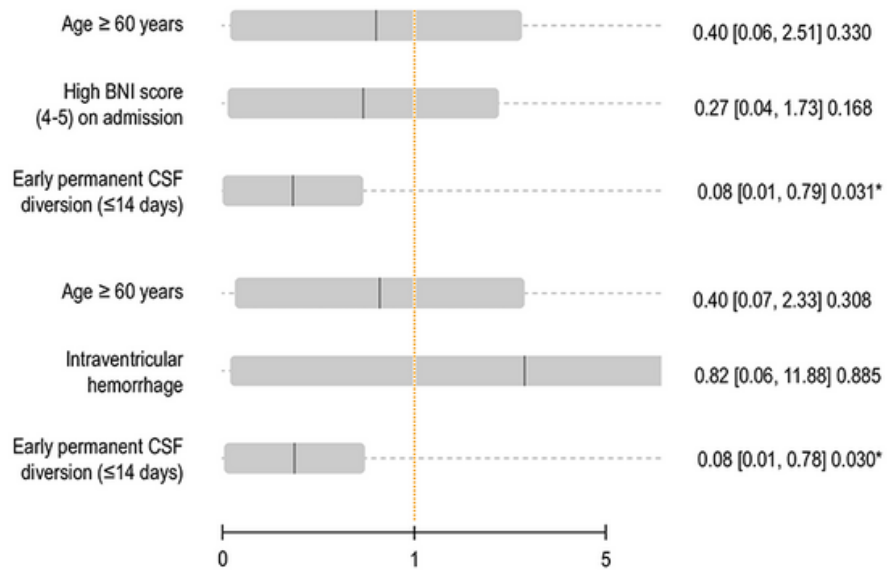

\section{Figure 2}

Multivariable covariate logistic regression analysis for "early permanent CSF diversion". C and D: Multivariable covariate logistic regression analysis for the primary endpoint variable "EVD-associated infection". Initial blood clot burden is represented by the "Barrow Neurological Institute (BNI) scale" dichotomized into "high BNI score" (BNI 4-5) vs. "low BNI score" (BNI 1-3), and presence of intraventricular hemorrhage in computed tomography on admission, included as covariate in a separate model each. EVD-days in situ and late permanent CSF diversion were identified as not being mutually exclusive and were hence analyzed separately. The binary logistic regression model includes the following covariates: age $\geq 60$ years (reference level: age $<60$ years), high BNI-score (reference level: BNI score 1-3), intraventricular hemorrhage (reference level: absence), duration of EVD (continuous variable) and late permanent CSF diversion (reference level: early permanent CSF diversion within the first 14 days). Statistical significance was set at alpha level of $P=.05$. Significance is indicated as follows: * $(p$ $\leq .05), * \star(p \leq .01)$. 
Age $\geq 60$ years

High BNI score (4-5) on admission

Early permanent CSF diversion ( $\leq 14$ days)

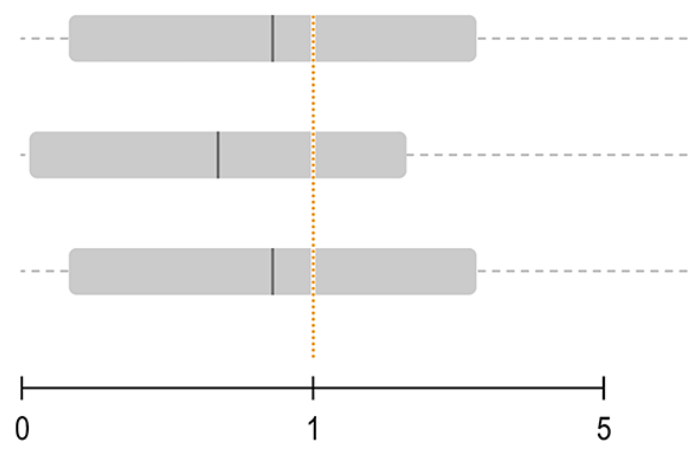

b

Delayed Cerebral Infarction

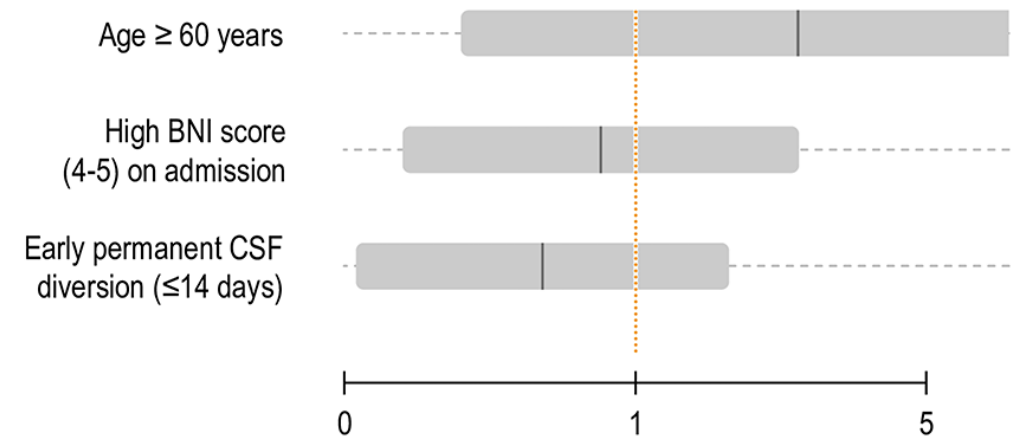

C Poor mRS (4-6) at discharge

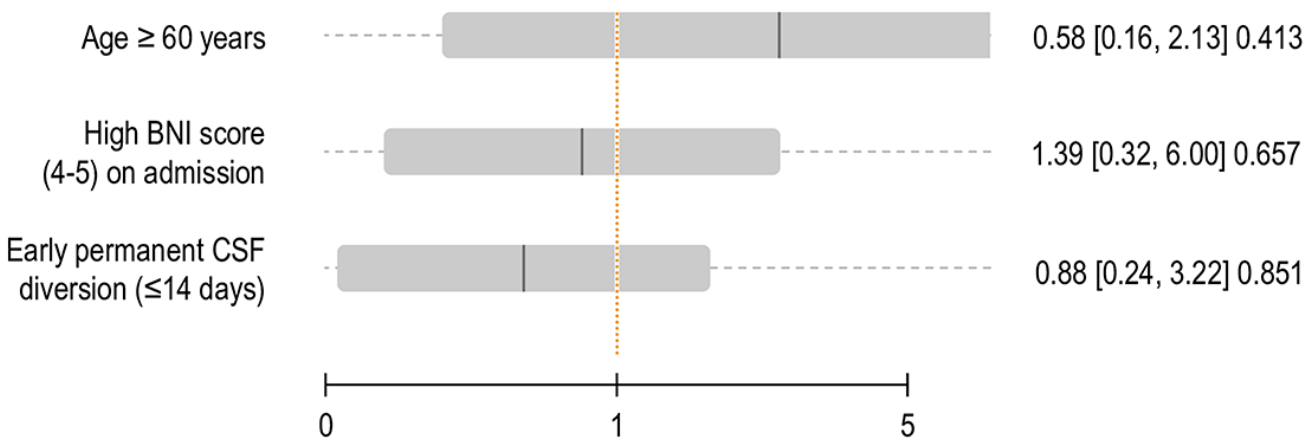

\section{Figure 3}

Multivariable covariate logistic regression analysis for the secondary endpoint variables A: "delayed cerebral vasospasm”, B: "delayed cortical infarction” and C: "poor modified Ranking Scale”. The Barrow Neurological Institute (BNI) score in computed tomography on admission represents the initial blood clot burden. The binary logistic regression model includes the following covariates: age $\geq 60$ years (reference level: age <60 years), high BNI-score (reference level: BNI score 1-3) and late permanent CSF diversion 
(reference level: early permanent CSF diversion within the first 14 days). Statistical significance was set at alpha level of $p=.05$. Significance is indicated as follows: * $(p \leq .05), \star \star(p \leq .01)$.

\section{Supplementary Files}

This is a list of supplementary files associated with this preprint. Click to download.

- Additionalfile1.docx 